\title{
Automation of S-parameters measurements of high-power microwave transistors in a contact device with tunable strip matching circuits
}

\author{
Vladimir Evseev ${ }^{1}$, Mikhail Ivlev $^{2}$, Elena Lupanova ${ }^{2}$, Sergey Nikulin ${ }^{1,2, *}$, Vitaliy Petrov ${ }^{2}$, and \\ Andrey Terentyev ${ }^{3}$ \\ ${ }^{1}$ Arzamas Engineering Design Bureau LLC, 607220 Arzamas (Nizhny Novgorod region), Russia \\ ${ }^{2}$ Alekseev Nizhny Novgorod State Technical University, 603950 Nizhny Novgorod, Russia \\ ${ }^{3}$ Rapid Telecom Systems Labs LLC, 603104 Nizhny Novgorod, Russia
}

\begin{abstract}
In the practice by microwave power transistor amplifiers developing, the variable load method is usually used to determine the impedances of matching circuits in the complex conjugate matching mode. This solution involves the use of expensive equipment - coaxial impedance tuners and contact devices for mounting transistors in low impedance strip lines. An even more complicated and expensive way is the concept of Xparameters, based on the use of unique measuring equipment - a non-linear vector network analyzer, and a simulator for non-linear circuits design. The article proposes an alternative solution adapted to the operation of the transistor in real conditions and allowing to design the output stages of microwave power amplifiers using analysis and optimization of linear electrical circuits. The essence of the proposed solution is to automate the measurement of non-linear S-parameters of high-power microwave transistors in a contact device with tunable strip matching circuits for various DC supply voltage, frequency and input power mode in case of continuous or pulse input signal. The nonlinear S-parameters of the contact device are measured using the method of spatially remote variable load in the frequency range, in which the line conditioning and the maximum output power are achieved. The minimum of the reflected wave amplitude and the maximum gain are reached using movable strip matching transformers. The S-parameters measured in the coaxial line are automatically recalculated to the physical boundaries of the transistor by registering the positions of the input and output strip transformers.
\end{abstract}

\section{Automated system for S-parameters measurements of microwave transistors in range of frequency and input powers}

It is preferable to measure the parameters of high-power microwave transistors using an automated system consisting of a vector network analyzer (VNA), a measuring module, a contact device with tunable strip matching circuits, a microwave power meter with an

*Corresponding author: nikulin-serg2006@yandex.ru 
attenuator, and power sources. The measuring system is based on the method of spatially remote variable load $[1,2]$.

The essence of the method is illustrated in Figure 1, which presents a simplified scheme of the implementation of the proposed method. The measuring module here is represented only by a spatially remote variable load, consisting of electrically long coaxial cable, directional coupler, $6 \mathrm{~dB}$-attenuator and variable load $\mathrm{Z}_{\mathrm{m}}$. The load accepts one of the following states: "Open" $(\mathrm{m}=1)$, "Short" $(\mathrm{m}=2)$ and "Matched" $(\mathrm{m}=3)$. To measure nonlinear S-parameters, any pair of states is sufficient, for example $\mathrm{m}=1,2$.

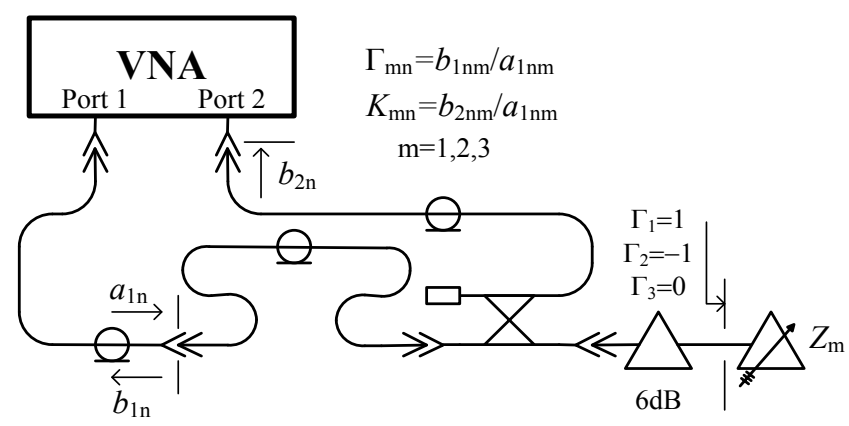

a)

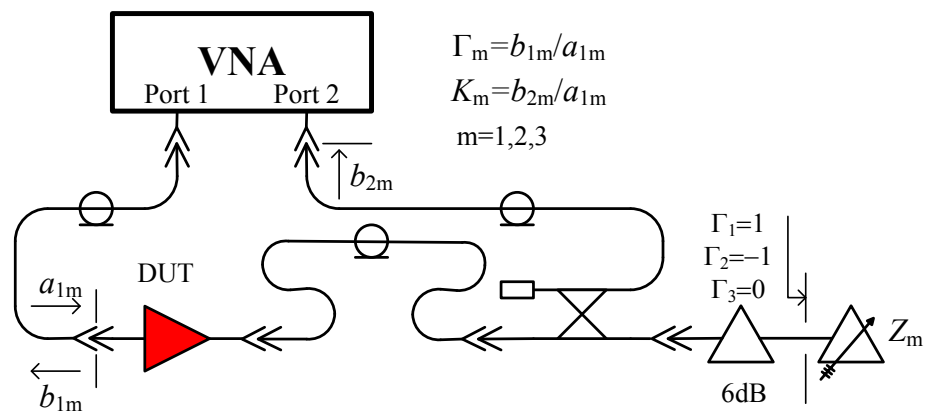

b)

Fig. 1. Simplified scheme for nonlinear S-parameters measuring of a contact device in a coaxial line: $a$ - calibration process; $b$ - measurement process.

First, the measurement module is connected directly to the first and second ports of the network analyzer, then the analyzed device is connected between the first port and the load. Network Analyzer measures the reflection coefficients $S_{11} \rightarrow \Gamma_{1 \mathrm{n}}, \Gamma_{2 \mathrm{n}}, \rightarrow \Gamma_{1}, \Gamma_{2}$ and the transmission coefficients $\mathrm{S}_{21} \rightarrow K_{1 \mathrm{n}}, K_{2 \mathrm{n}}, \rightarrow K_{1}, K_{2}$. Nonlinear S-parameters of the analyzed device is obtained from the following relations:

$$
\begin{gathered}
S_{11}^{d u t}=\frac{\Gamma_{1} K_{2} \Gamma_{2 n} / K_{2 n}-\Gamma_{2} K_{1} \Gamma_{1 n} / K_{1 n}}{K_{2} \Gamma_{2 n} / K_{2 n}-K_{1} \Gamma_{1 n} / K_{1 n}}, \quad S_{12}^{d u t}=\frac{\Gamma_{2}-\Gamma_{1}}{K_{2} \Gamma_{2 n} / K_{2 n}-K_{1} \Gamma_{1 n} / K_{1 n}}, \\
S_{21}^{d u t}=\frac{K_{1} K_{2}\left(\Gamma_{2 n}-\Gamma_{1 n}\right)}{K_{1 n} K_{2} \Gamma_{2 n}-K_{2 n} K_{1} \Gamma_{1 n}}, \quad S_{22}^{d u t}=\frac{K_{1 n} K_{2}-K_{2 n} K_{1}}{K_{1 n} K_{2} \Gamma_{2 n}-K_{2 n} K_{1} \Gamma_{1 n}} .
\end{gathered}
$$

The architecture of the measuring module is determined by the network analyzer. In addition to the spatially remote load, two directional couplers and a microwave power amplifier in the measuring module are used to obtain a high-level probe signal. When using a network analyzer with access to measuring receivers, the connection diagram of these elements with each other is well known [3]. In case of a common two-port VNA, or 
modular analyzer with the function of reflection coefficient and transmission coefficient measurement, it is recommended to use the connection shown in Figure 2.

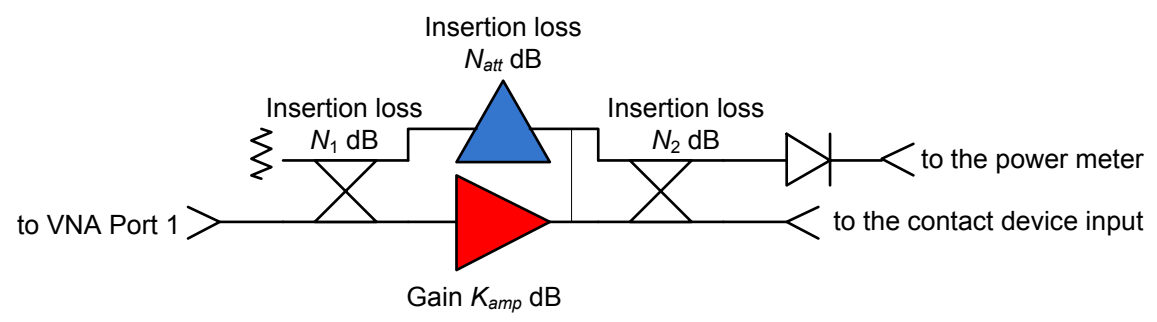

Fig. 2. The scheme of the high-level probe signal conditioning.

As shown in Figure 2, an attenuator with attenuation $\mathrm{N}_{\text {att }}$ is introduced into the probe signal conditioning circuit. The value of $\mathrm{N}_{\text {att }}$ should be chosen from the next condition:

$$
K_{a m p}+\left(N_{1}+N_{a t t}+N_{2}\right) \approx 0 d B .
$$

When measuring non-linear S-parameters in the pulse mode of the transistor, a pulse modulator should be added to the scheme.

The measurement system software performs the following functions:

- measurement of the reflection coefficients from a spatially remote variable load;

- OSM calibration of the first port at different input signal power levels;

- $\quad$ power calibration of the input signal;

- measurement of reflection coefficients and transmission coefficients of the remote variable load;

- measurement of reflection coefficients and transmission coefficients of a contact device with a remote variable load in predetermined frequency range in the mode, optimized for best matching and maximum gain;

- calculation of non-linear S-parameters of the contact device and transfer of the results to the physical boundaries of the transistor;

- formation of a library of s2p-models of high-power microwave transistors [4].

The fundamental tone scattering parameters of transistors depend on the amplitude of the input signal and the load impedance. To design the powerful output amplifier, it is enough to have the S-parameters in the matching mode of the transistor - such S-parameters for various amplitudes of continuous and pulsed signals and at various DC supply voltage can be determined using the proposed automated system.

The appearance of the experimental sample of an automated measuring system with a VNA Planar 804/1 is shown in Figure 3.

\section{Universal contact device with tunable strip matching circuits}

The idea of constructing a contact device with tunable matching circuits was discussed in [3] and consists in the use of strip transformers installed on sufficiently long segments of shielded strip transmission lines, in the gap of which the measured transistor is placed. The impedance transformation effect is realized using quarter-wave transformers in the form of a metal plate with a dielectric film that insulates it from strip conductors. Due to the introduction of additional capacitance between the strip conductor and the screen placed on the upper surface of the board, the impedance transformer lowers the line impedance at the installation site. Figure $4 \mathrm{a}$ illustrates the principle of operation of the transformer, and Figure $4 \mathrm{~b}$ illustrates its design. As a dielectric, a teflon film with a thickness of $20 \mu \mathrm{m}$ was used. The small film thickness makes it possible to obtain a wave impedance of the 
transformer of less than $10 \mathrm{Ohms}$. It ensures the transformation of the impedance of the signal source and load at the physical boundaries of the transistor to values of the order of units of Ohms. The transformer moves along the transmission line to adjust the matching of the transistor.

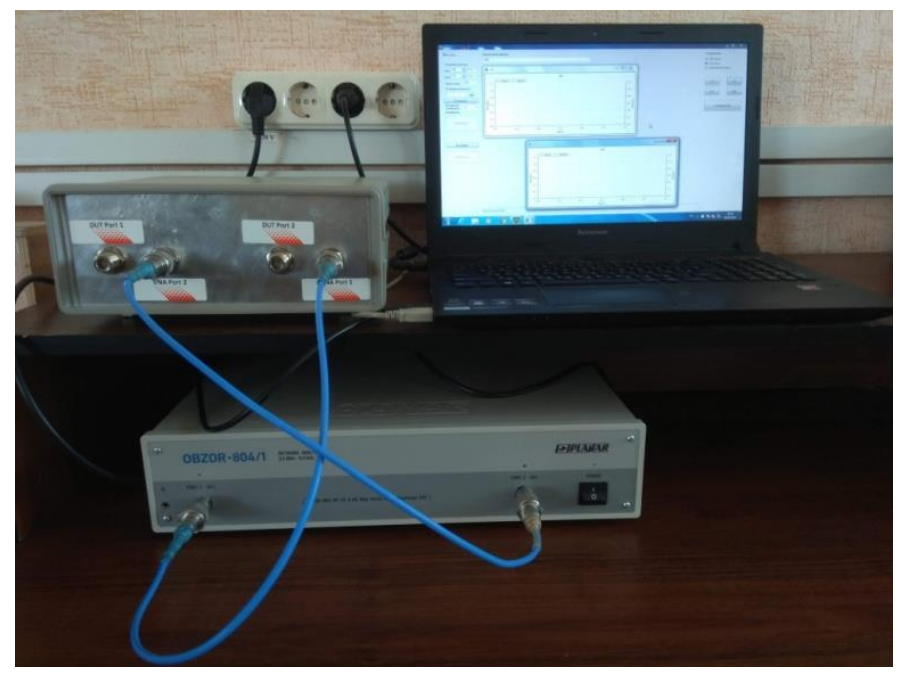

Fig. 3. Appearance of an automated measuring system with a network analyzer Planar -804/1.

By installing the input and output transformers at optimal distances, it is possible not only to create low impedances at the input or output of the transistor, but also to compensate its capacitances by the inductive component of the transforming circuits impedances. The matching in this way is obtained in a rather narrow frequency range; however, it is very simple to tune the device to a different frequency range. To carry out measurements in the new frequency range, it is enough to just move the transformer plates and select their new position with respect to the transistor, providing acceptable matching at the input and the maximum value of gain of the measuring device.

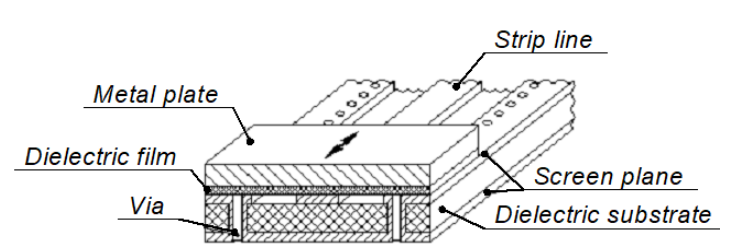

a)

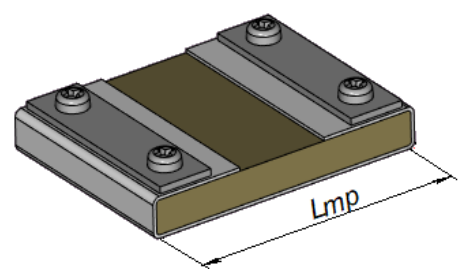

b)

Fig. 4. Impedance transformer: $a$ - principle of operation; $b$-construction of movable element.

A fundamental feature of the proposed tooling, shown in Figure 5, is its versatility and the ability to determine the S-parameters of a transistor relative to its physical boundaries due to automatic registration of the position of matching devices. The parameters of transitions, lines and transformers are determined beforehand during the calibration process. The design is adapted to various types and sizes of transistor cases by replacing only two parts.

Figure 6 shows a scheme of the contact device. It contains coaxial-to-microstrip transitions X1 and X2 for connection the device to a standard coaxial line. On segments of an asymmetric strip line, movable transforming chains $\mathrm{Z} 1$ and $\mathrm{Z} 2$ are installed. Between these segments, the measured transistor VT is connected. 


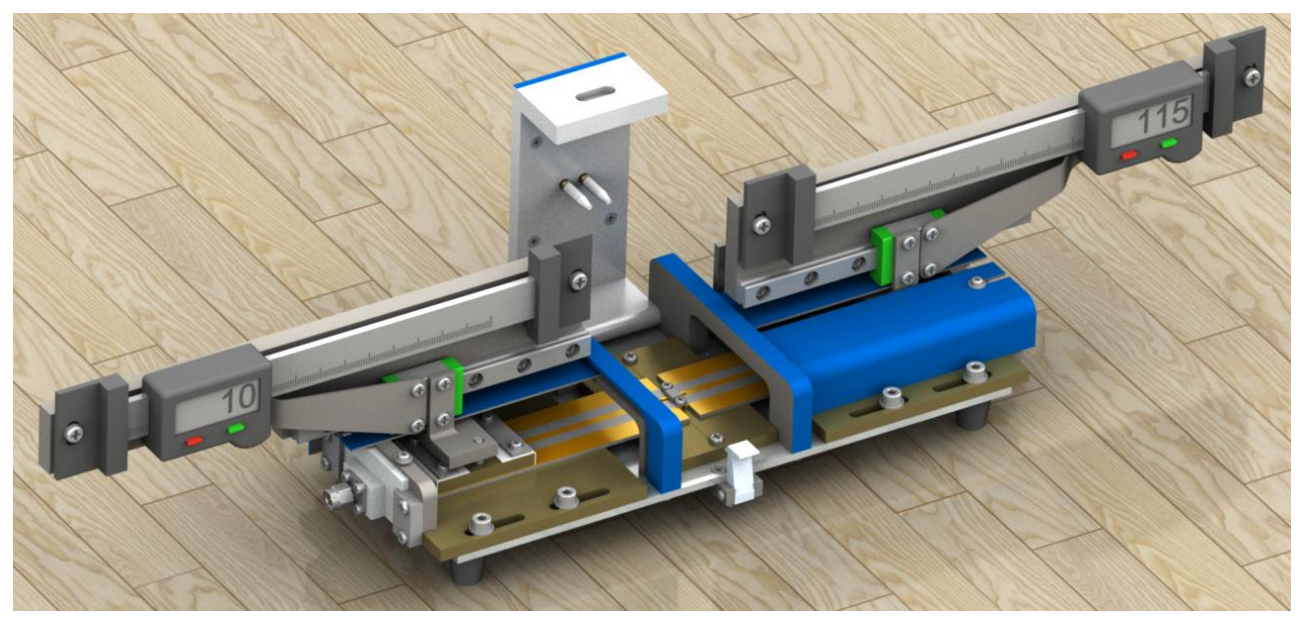

Fig. 5. The appearance of the contact device with tunable matching circuits.

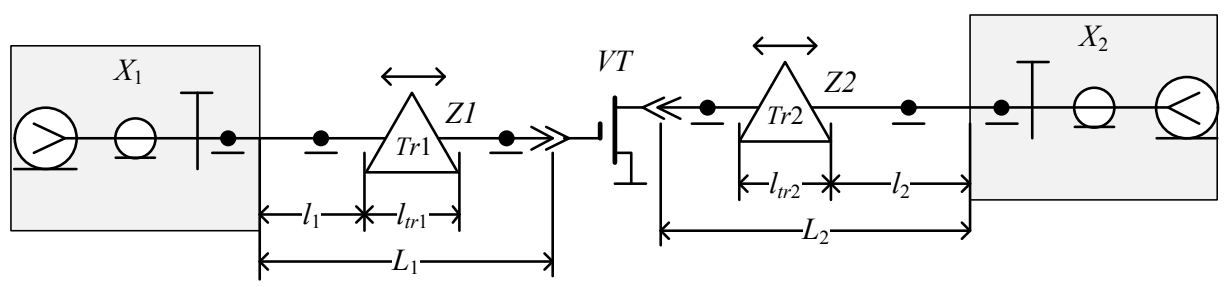

Fig. 6. Scheme of the contact device

To determine the S-parameters of the transistor relative to its physical boundaries, a standard procedure is used to exclude the input and output circuits surrounding the transistor from the measurement results. Files of the s2p format, $S_{i k}^{a}$ and $S_{i k}^{b}$, for each of these circuits are formed on the basis of a cascade connection of the coaxial-to-microstrip transition, two segments of the strip line and an impedance transformer. For example, formulas for calculating the parameters of the output circuit are following:

$$
\begin{gathered}
S_{11}^{b}=S_{11}^{x 2}+\frac{S_{21}^{x 2} S_{12}^{x 2} S_{11}^{t r 2} \exp \left(-i 2 \gamma l_{2}\right)}{1-S_{22}^{x 2} S_{11}^{t r 2} \exp \left(-i 2 \gamma l_{2}\right)}, \\
S_{21}^{b}=\frac{S_{21}^{x 2} S_{21}^{t r 2} \exp \left[-i 2 \gamma\left(L_{2}-l_{t r 2}\right)\right]}{1-S_{22}^{x 2} S_{11}^{t r 2} \exp \left(-i 2 \gamma l_{2}\right)}, \\
S_{12}^{b}=\frac{S_{12}^{x 2} S_{12}^{t r 2} \exp \left[-i 2 \gamma\left(L_{2}-l_{t r 2}\right)\right]}{1-S_{22}^{x 2} S_{11}^{t r 2} \exp \left(-i 2 \gamma l_{2}\right)}, \\
S_{22}^{b}=S_{22}^{t r 2} \exp \left[-i 2 \gamma\left(L_{2}-l_{t r 2}-l_{2}\right)\right]+\frac{S_{21}^{t r 2} S_{12}^{t r 2} S_{22}^{x 2} \exp \left[-i 2 \gamma\left(L_{2}-l_{t r 2}\right)\right]}{1-S_{22}^{x 2} S_{11}^{t r 2} \exp \left(-i 2 \gamma l_{2}\right)},
\end{gathered}
$$

where $S_{i k}^{x 2}$ - S-parameters of coaxial-to-microstrip transition, $S_{i k}^{t r 2}$ - S-parameters of output transformer, $\gamma$ - propagation constant for microstrip line. 
The obtained measurement results allow to form the $s 2 p$ transistor models in a given frequency range for computer-aided design of amplifier modules using linear analysis and optimization $[3,4]$.

\section{Transistor protection device}

To prevent the damage of the measuring transistor, a protection device is introduced into the measuring amplifier. It performs the following functions:

- time delay of the supplying voltage to the drain of the investigated transistor after turning on the power sources;

- $\quad$ protection against exceeding the pre-set value of the drain current;

- $\quad$ switching off the voltage at the drain while reducing the bias on the shutter below the pre-set value.

The protection device is connected between external adjustable DC sources that form the drain voltage and the gate bias, and transistor power adapters (bias tees).

The protection efficiency is ensured by a short response time, not exceeding $8 \mu \mathrm{s}$.

\section{Conclusion}

The considered automated system makes it possible to measure nonlinear S-parameters of a contact device with tunable strip matching circuits in a coaxial channel using a spatially remote variable load method. Automatic registration of the position of strip matching transformers allows real-time determination of the S-parameters of high-power microwave transistors relative to their physical boundaries. It is significant that the proposed solution opens up unique opportunities for the formation of a library of $s 2 \mathrm{p}$-models of transistors, including double-gate transistors in the paraphase mode of operation, in the space of various power supply modes for DC supply voltage, frequency and power of a continuous or pulsed input signal.

\section{References}

1. S.M. Nikulin, A.I. Torgovanov, Measurement large signal nonlinear microwave circuit S-parameters using spatially remote load, Sensors \& Systems, 11, pp. 27-33 (2014)

2. S.M. Nikulin, A.I. Torgovanov, Microwave power amplifiers design. Efficiency of remote variable load method, Electronics: Science, Technology, Business, 3, pp. 60-65 (2015)

3. V.I. Evseev, E.A. Lupanova, A.B. Kulikov, S.M. Nikulin, V.V. Petrov, Measuring amplifiers and technology for determining large-signal S-parameters of microwave transistors, Papers of Microwave electronics conference, Saint-Peetersburg Electrotechnical University, part 1, pp. 282-286 (2018)

4. V.I. Evseev, A.A. Krylov, E.A. Lupanova, S.L. Morugin, S.M. Nikulin, V.V. Petrov, Creating of a library of $s 2 p$ models of high-power microwave transistors using computer-aided design or a full-scale experiment, Papers of Microwave electronics conference, Saint-Peetersburg Electrotechnical University, pp. 63-67 (2019) 